АНАЛИЗ ВЛИЯНИЯ УСЛОВИЙ ЗАКРЕПЛЕНИЯ НА СМЕЩЕНИЕ НУЛЯ КОРИОЛИСОВОГО РАСХОДОМЕРА

\author{
A.A. Яушев \\ Южно-Уральский государственный университет, г. Челябинск
}

Кориолисов расходомер предназначен для измерения массового расхода жидкостей и газов. Принцип действия расходомера основан на изменении фаз механических колебаний двух пар точек U-образных трубок, по которым движется измеряемая среда. Трубки под воздействием внешнего возбуждения совершают установившиеся вынужденные колебания на резонансной частоте. Сдвиг фаз пропорционален величине массового расхода. Поэтому изменения величины фазового сдвига, не связанные с изменением параметров потока измеряемой среды, приводят к увеличению погрешности измерения массового расхода. Статья посвящена экспериментальной оценке влияния условий закрепления кориолисового расходомера на смещение нуля. Смещение нуля кориолисового расходомера это величина разности фаз при отсутствии течения измеряемой среды. Для исследования влияния условий закрепления на смещение нуля разработана и изготовлена специальная оснастка, позволяющая изменять жесткость трубопроводов, к которым присоединяется расходомер, за счет изменения длин трубопроводов. Изменение длины трубопроводов приводит к изменению форм и частот собственных колебаний системы «расходомер трубопроводы». Для определения форм и частот собственных колебаний системы «расходомер - трубопроводы» проведены модальные испытания кориолисового расходомера при разных длинах трубопроводов. Испытания проведены на расходомере со стоячей водой и при текущей воде с постоянным расходом. Установлено, что максимальное смещение нуля наблюдается при длинах трубопроводов, на которых частота собственных колебаний связанной системы «расходомер - трубопроводы» совпадает с частотой резонансных колебаний трубок, на которой работает расходомер. Для уменьшения влияния длин трубопровода на смещение нуля в статье рассмотрено дополнительное жесткое закрепление расходомера за корпус.

Ключевые слова: кориолисов расходомер, смещение нуля, экспериментальный модальнылй анализ.

Кориолисов расходомер предназначен для измерения массового расхода жидкостей и газов. Обзор конструкций кориолисовых расходомеров приведен в [1-3]. Расходомер, представленный на рис. 1 , состоит из основания 1 , корпуса 2 и двух U-образных колеблющихся трубок 3 , по которым течет измеряемая среда. Трубки под действием задающей катушки 4 совершают установившиеся вынужденные колебания на резонансной частоте. Форма установившихся вынужденных

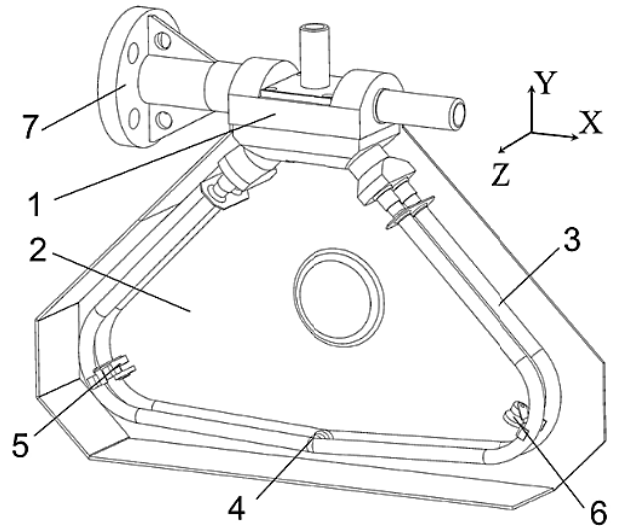

Рис. 1. Кориолисов расходомер: 1 - основание; 2 - корпус; 3 - трубки; 4 - задающая катушка; 5, 6 - измерительные катушки; 7 - фланец колебаний представляет собой движение трубок в противоположном друг к другу направлении из плоскости XY. Поступательное движение среды во вращательном движении трубок приводит к возникновению кориолисового ускорения и, соответственно, кориолисовой силы. Из-за действия кориолисовой силы жидкости на трубки происходит сдвиг фаз механических колебаний двух трубок в местах установки измерительных катушек 5 и 6 , пропорциональный массовому расходу. Подробное описание принципа работы кориолисового расходомера приведено в [4].

В кориолисовых расходомерах массовый расход определяют с помощью измерения сдвига фаз сигналов измерительных катушек. Поэтому изменения величины фазового сдвига, не связанные 


\section{Контроль и испытания}

с потоком измеряемой среды, приводят к увеличению погрешности измерения массового расхода.

Смещение нуля кориолисового расходомера - это величина разности фаз при отсутствии потока измеряемой среды. Обычно оно примерно в 1000 раз меньше, чем полезный фазовый сдвиг при номинальном расходе. Однако величина смещения нуля существенна при точности датчика $0,1 \%$. Каждый прибор характеризуется смещением нуля, величина которого индивидуальна и определяется на этапе изготовления прибора.

Одна из особенностей кориолисового расходомера состоит в том, что присоединение расходомера к трубопроводам на месте эксплуатации может приводить к смещению нуля. В этом случае за смещение нуля принимают его значение после установки. Реже, но намного опаснее возникновение ситуации нестабильного смещения нуля установленного расходомера, когда оно меняется с течением времени, что приводит к неконтролируемому росту погрешности измерения массового расхода.

Анализу причин возникновения смещения нуля в кориолисовом расходомере посвящены работы [5-8]. С помощью прямотрубной модели кориолисового расходомера, расчетным путем, оценивается влияние на смещения нуля в статьях $[5,6]$ неравномерной жесткости и массы трубки, демпфирования, а в [7] несимметричное расположение катушки возбуждения и катушек измерения. В статье [8] экспериментальным путем исследуется влияние добавления точечных масс на трубки и изменение демпфирования трубок на смещение нуля кориолисового расходомера с изогнутыми трубками. В существующих работах проблеме смещения нуля из-за неправильной установки расходомера уделяется недостаточное внимание. В этой статье рассматривается влияние условий закрепления кориолисового расходомера на смещение нуля.

\section{Модальные испытания}

Для исследования влияния различных условий закрепления на смещение нуля разработана и изготовлена специальная оснастка, позволяющая изменять жесткость трубопроводов за счет изменения их длины. Оснастка представлена на рис. 2. Она состоит из основания 1, представляющего собой швеллер, и двух трубопроводов 2 с приваренными на одном конце фланцами. К фланцам на трубопроводах с помощью болтов присоединяется расходомер 3. Трубопроводы закрепляются с помощью двух неподвижных зажимов 4 на краях приспособления. По направляющим 6 перемещаются подвижные зажимы 5. С помощью двух подвижных зажимов изменяются длины трубопроводов $\mathrm{L}_{1}$ и $\mathrm{L}_{2}$. Оснастка закреплена на тяжелой и жесткой плите.

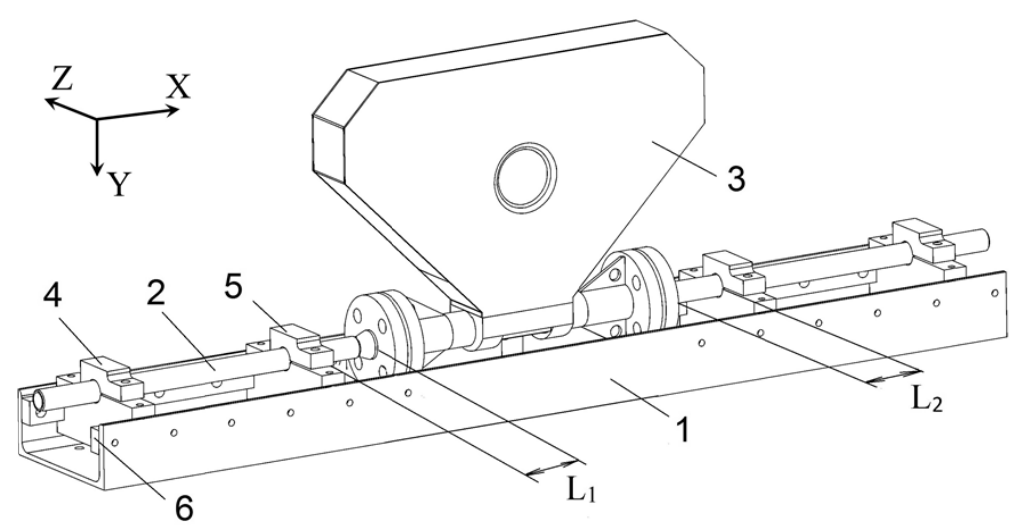

Рис. 2. Схема оснастки для закрепления расходомера: 1 - основание; 2 - трубопровод; 3 - расходомер; 4 - неподвижный зажим; 5 - подвижный зажим; 6 - направляющая

$\mathrm{C}$ использованием оснастки получена оценка влияния длин трубопроводов $\mathrm{L}_{1}$ и $\mathrm{L}_{2}$, к которым крепится расходомер, на смещения нуля. Изменение длины трубопроводов влияет на формы и частоты собственных колебаний связанной системы «расходомер - трубопроводы». Чтобы отслеживать изменение этих форм и частот, проведены модальные испытания системы «расходомер - трубопроводы» при различных длинах трубопроводов.

Модальные испытания заполненного водой расходомера, присоединенного к трубопроводам, выполнены при выключенной электронике (отсутствии возбуждения на рабочей частоте). Найдены длины, на которых частоты собственных колебаний связанной системы «расходомер - трубо- 
проводы» сближаются с частотой резонансных колебаний трубок, на которой работает расходомер. При всех длинах трубопроводов после модальных испытаний с помощью штатной электроники измерено смещение нуля.

Модальные испытания расходомера [9, 10] выполнены с использованием программноаппаратного комплекса, в состав которого входят:

- 56-канальная измерительная система LMS SCADAS LAB для сбора и обработки динамических сигналов;

- модуль Impact Testing пакета LMS Test.Lab для проведения модальных испытаний при ударном возбуждении и последующей идентификации мод колебаний;

- ударный молоток РСВ 086С03 со встроенным датчиком силы;

- трехкомпонентные пьезоакселерометры РСВ 356А32 чувствительностью $100 \mathrm{mV} / \mathrm{g}$.

Возбуждение колебаний осуществлялось с помощью модального молотка последовательно в восьми точках на корпусе расходомера и трубопроводах (рис. 3) по трем направлениям (X, Y, Z).

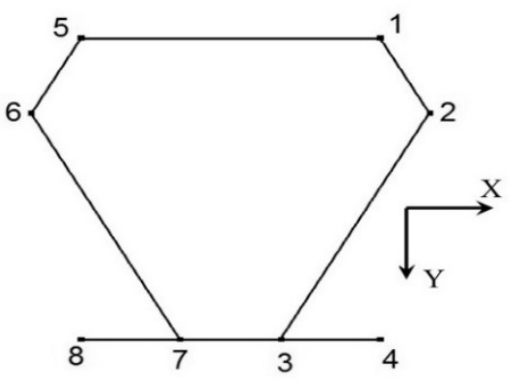

Рис. 3. Экспериментальная модель корпуса расходомера: точки 1-8 места возбуждения колебаний; точки 1, 5, 7 - места, где установлены акселерометры

Отклик измерен с помощью трехкомпонентных акселерометров, расположенных в трех точках на корпусе (точки 1, 5,7 на рис. 3). Для определения собственных форм колебаний корпуса в программе LMS Test.Lab построена каркасная модель. Узлы этой модели совпадают с точками, в которых установлены акселерометры и где проводилось возбуждение колебаний.

При возбуждении колебаний молотком в выбранную точку конструкции нанесены последовательно несколько ударов. По результатам каждого удара измерен входной ударный импульс и отклики по виброускорениям во всех измерительных точках конструкции и получены частотные характеристики FRF (frequency response function) [11, 12]. Далее эксперимент повторялся при возбуждении в другой точке. По полученным осредненным частотным характеристикам с помощью алгоритма PolyMax $[13,14]$ идентифицированы формы и частоты собственных колебаний системы «расходомер трубопроводы».

В спектре отклика на ударное воздействие присутствует множество пиков, рассмотрены только три, которые при изменении длины трубопроводов от 20 до 250 мм сближаются с рабочей частотой резонансных колебаний трубок. Три формы собственных колебаний системы «расходомер - трубопроводы» представлены на рис. 4. Форма 1 и 2 соответствует колебаниям системы «расходомер - трубопроводы» из плоскости XY. Форма 3 соответствует колебаниям в плоскости XY.

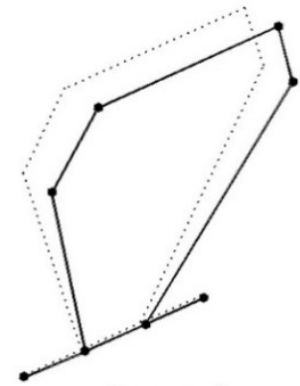

Форма 1

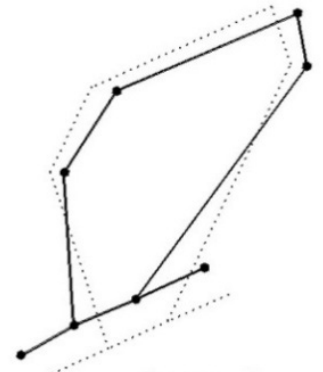

Форма 2

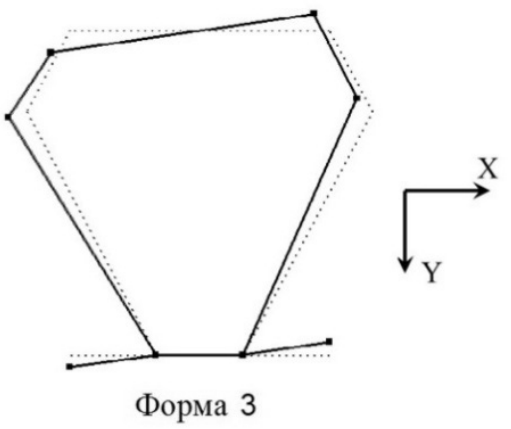

Форма 3

Рис. 4. Формы собственных колебаний корпуса расходомера

В табл. 1 приведены формы и частоты собственных колебаний системы «расходомер - трубопроводы» и смещения нуля при разных длинах трубопроводов. Длина трубопроводов слева и справа одинаковая $\mathrm{L}_{1}=\mathrm{L}_{2}$.

Для расходомера с трубками, заполненными стоячей водой, на рис. 5 представлена зависимость смещения нуля от длин трубопроводов при равенстве длин трубопроводов слева и справа $\mathrm{L}_{1}=\mathrm{L}_{2}$. Экспериментально установлено, что существуют длины трубопроводов, на которых на- 


\section{Контроль и испытания}

блюдается резкое увеличение смещения нуля. Как показал анализ, максимальное смещение нуля соответствует длинам трубопроводов, при которых частоты собственных колебаний системы «расходомер - трубопроводы» сближаются с рабочей частотой резонансных колебаний трубок. Установлено, что изменение длины трубопровода в одну или другую сторону на 5-10 мм приводит к восстановлению нуля до начального значения.

На практике это означает, что если частота собственных колебаний системы «расходомер трубопроводы» близка с рабочей частотой резонансных колебаний трубок, то смещение нуля может меняться со временем. Это связано в первую очередь с зависимостью рабочей частоты резонансных колебаний трубок от плотности текущей внутри трубки среды. При изменении плотности (например, из-за изменения температуры) рабочая частота резонансных колебаний трубок меняется намного сильнее чем частоты собственных колебаний системы «расходомер - трубопроводы». Таким образом, смещение нуля становится не контролируемым.

Таблица 1

Смещения нуля и частоты собственных колебаний системы «расходомер - трубопроводы» с трубками, заполненными водой при разных длинах трубопроводов

\begin{tabular}{|l|c|c|c|c|c|c|}
\hline \multirow{2}{*}{ Характеристика } & \multicolumn{7}{|c|}{ Длины трубопроводов $\mathrm{L}_{1}=\mathrm{L}_{2}$, мм } \\
\cline { 2 - 7 } & 24 & 50 & 100 & 150 & 202 & 211 \\
\hline $\begin{array}{l}\text { Рабочая частота } \\
\text { расходомера, Гц }\end{array}$ & $\mathbf{9 4 , 1}$ & 94,1 & 94,1 & 94,1 & $\mathbf{9 4 , 1}$ & $\mathbf{9 4 , 1}$ \\
\hline Частота формы 1, Гц & $\mathbf{9 4}$ & 91,8 & 89 & 87,7 & 86,6 & 86,4 \\
\hline Частота формы 2, Гц & 262 & 207,8 & 168,5 & 123,8 & $\mathbf{9 4 , 5}$ & 93,6 \\
\hline Частота формы 3, Гц & 102,5 & 102,1 & 101,2 & 99,3 & 95,7 & $\mathbf{9 4 , 2}$ \\
\hline \multicolumn{7}{|c|}{ Стоячая вода } \\
\hline Смещение нуля, 10 $0^{-6} \mathrm{c}$ & $-0,24$ & 0,014 & 0,014 & 0,014 & $-0,83$ & $-0,25$ \\
\hline \multicolumn{7}{|}{ Постоянный массовый расход 1,5 түч } \\
\hline Сдвиг фаз, $10^{-6}$ с & 14,27 & 14,52 & 14,52 & 14,52 & 13,7 & 14,27 \\
\hline
\end{tabular}

Если длины трубопроводов слева и справа не равны $\mathrm{L}_{1} \neq \mathrm{L}_{2}$, сближение частот собственных колебаний системы «расходомер - трубопроводы» с рабочей частотой резонансных колебания трубок будет приводить к смещению нуля так же, как и при одинаковых длинах трубопроводов.

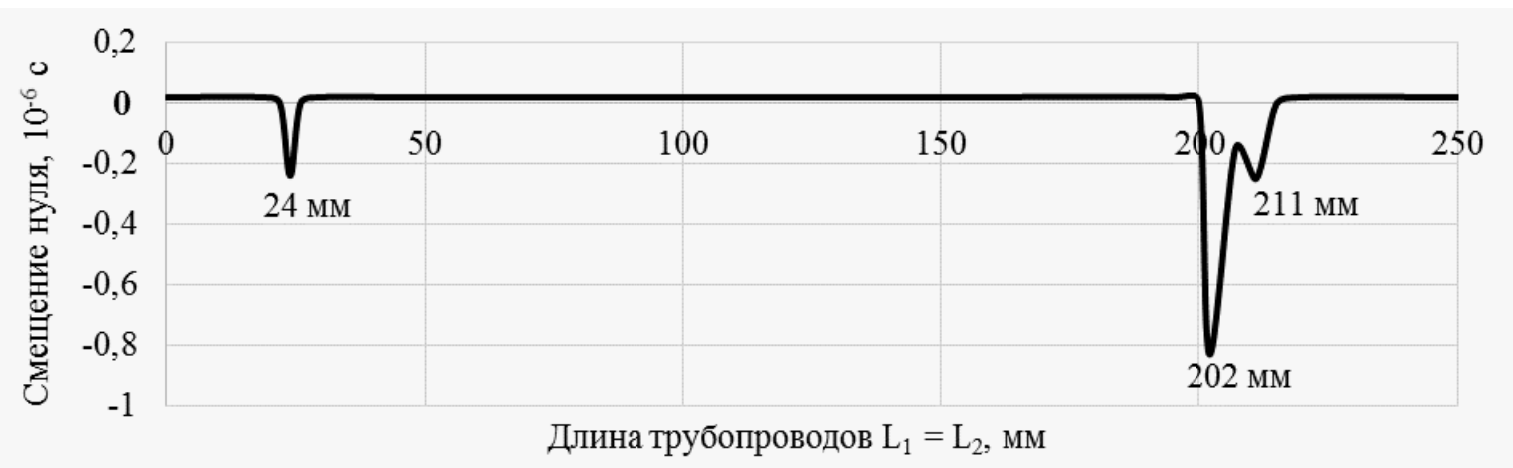

Рис. 5. Зависимость смещения нуля от длины трубопровода для расходомера со стоячей водой

На рабочем режиме кориолисового расходомера измеряемая среда движется. Поэтому выполнена экспериментальная оценка влияния условий закрепления кориолисового расходомера на погрешность измерения расхода в случае текущей воды. Для этого использован проливочный стенд, позволяющий создавать поток воды с постоянным расходом (1,5 т/ч), равным половине номинального расхода расходомера. Для контроля расхода воды использован эталонный расходомер, подсоединенный последовательно перед исследуемым расходомером (рис. 6). Жесткость закрепления эталонного расходомера постоянна, а у исследуемого расходомера меняется длина присоединенных трубопроводов. В табл. 1 приведен сдвиг фаз исследуемого расходомера при разных длинах трубопроводов. Показания эталонного расходомера при изменении длин трубопроводов не меняются. 
Из табл. 1 и рис. 7 видно, что сдвиг фаз при расходе воды 1,5 т/ч составляет 14,52 мкс при всех длинах трубопровода кроме длин, при которых частоты собственных колебаний системы «расходомер - трубопроводы» сближаются с рабочей частотой резонансных колебаний трубок. Сближение с рабочей частотой приводит к изменению сдвига фаз до 13,7 мкс. Это изменение сдвига фаз $(14,52-13,7=0,82)$ почти равно смещению нуля при отсутствии потока и при той же длине трубопроводов. Таким образом, для оценки влияния условий закрепления рассматриваемых кориолисовых расходомеров на точность измерения расхода достаточно оценивать смещение нуля при отсутствии потока.

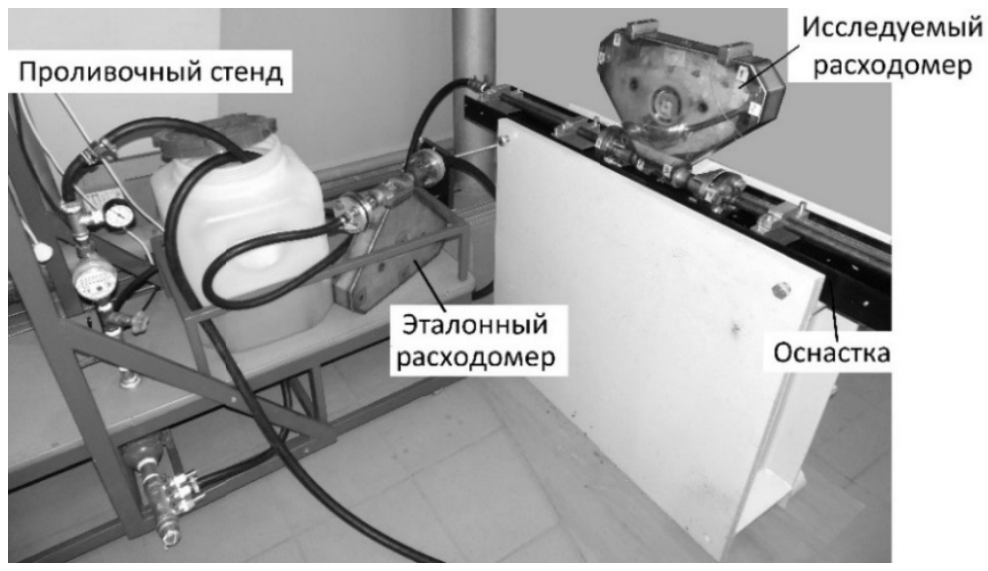

Рис. 6. Экспериментальный стенд

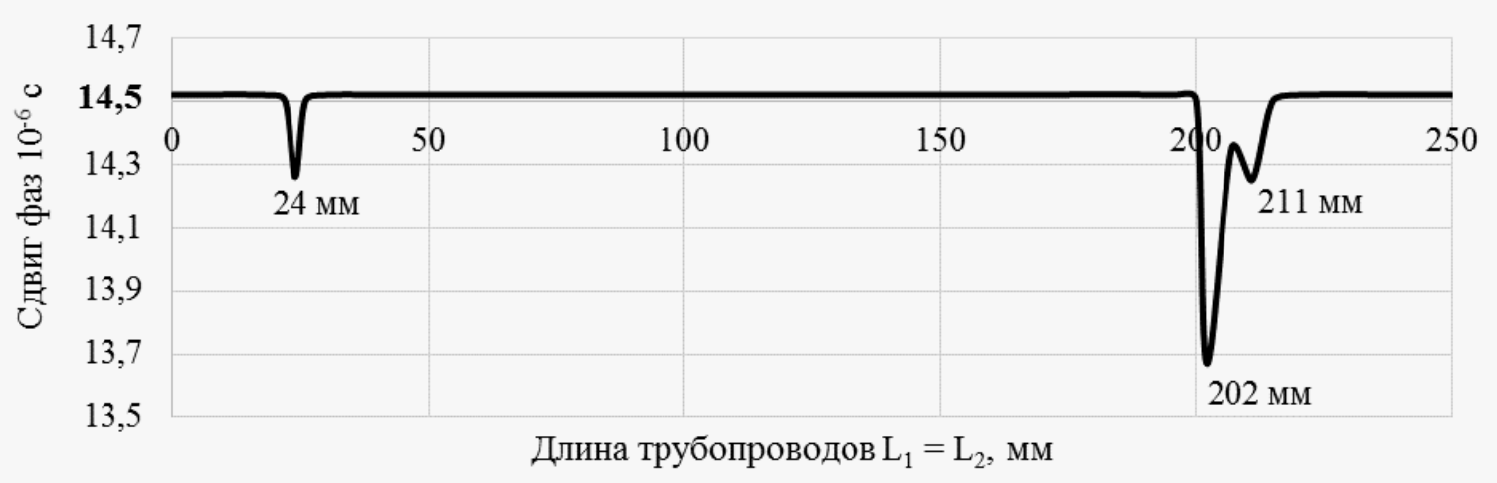

Рис. 7. Зависимость сдвига фаз от длины трубопровода при постоянном расходе воды 1,5 т/4

\section{Закрепление расходомера за корпус}

Одним из возможных способов уменьшения влияния длины трубопроводов на смешение нуля кориолисового расходомера может быть дополнительное закрепление расходомера. Оно позволяет уменьшить количество форм собственных колебаний системы «расходомер - трубопроводы», частоты которых могут совпасть с рабочей частотой резонансных колебаний расходомера.

Рассмотрено жесткое закрепление расходомера за корпус. На верхний бортик корпуса расходомера приварены два кронштейна. Кронштейны крепятся к жесткому основанию (рис. 8).

При дополнительном закреплении существует только одна форма собственных колебаний системы «расходомер - трубопроводы», частота которой сближается с рабочей частотой колебаний трубок (рис. 9). Рассмотрены длины трубопроводов до 250 мм.

В табл. 2 приведены смещения нуля и частоты собственных колебаний системы «расходомер трубопроводы» с трубками со стоячей водой и дополнительным жестким закреплением расходомера за корпус при разных длинах трубопроводов.

На рис. 10 представлена зависимость смещения нуля от длин трубопроводов в случае дополнительного закрепления расходомера за корпус. Максимальное смещение нуля соответствует длине трубопровода, при которой одна из частот собственных колебаний системы «расходомер трубопроводы» сближается с рабочей частотой резонансных колебаний трубок. 


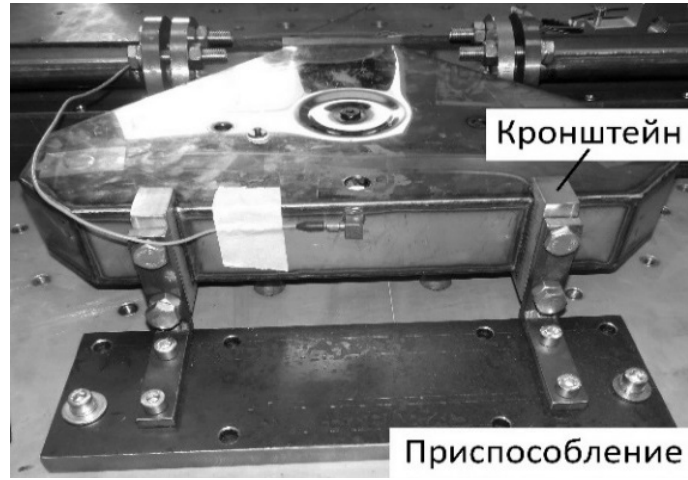

Рис. 8. Расходомер, закрепленный за корпус

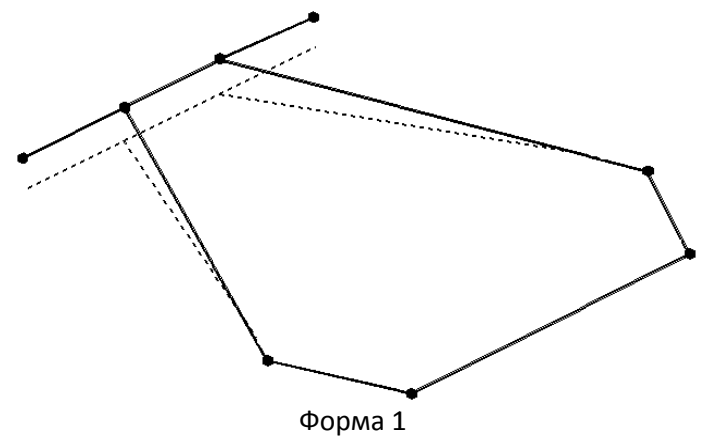

Рис. 9. Формы собственных колебаний системы «расходомер - трубопроводы»

Таким образом, дополнительное жесткое закрепление расходомера за корпус позволяет уменьшить вероятность недопустимого смещения нуля из-за неправильного выбора длин трубопроводов при установке расходомера на месте его эксплуатации.

Таблица 2

Смещения нуля и частоты собственных колебаний системы «расходомер - трубопроводы» с трубками со стоячей водой и дополнительным жестким закреплением расходомера за корпус при разных длинах трубопроводов

\begin{tabular}{|l|c|c|c|c|c|c|}
\hline \multirow{2}{*}{ Характеристика } & \multicolumn{7}{|c|}{ Длины трубопроводов $\mathrm{L}_{1}=\mathrm{L}_{2}$, мм } \\
\cline { 2 - 8 } & 20 & 50 & 100 & 150 & 179 & 250 \\
\hline Рабочая частота расходомера, Гц & 94,1 & 94,1 & 94,1 & 94,1 & $\mathbf{9 4 , 1}$ & 94,1 \\
\hline Частота формы 1, Гц & 205 & 191 & 150 & 114 & $\mathbf{9 4}$ & 73 \\
\hline Смещение нуля, $10^{-6} \mathrm{c}$ & 0,02 & 0,02 & 0,02 & 0,02 & $-0,16$ & 0,02 \\
\hline
\end{tabular}

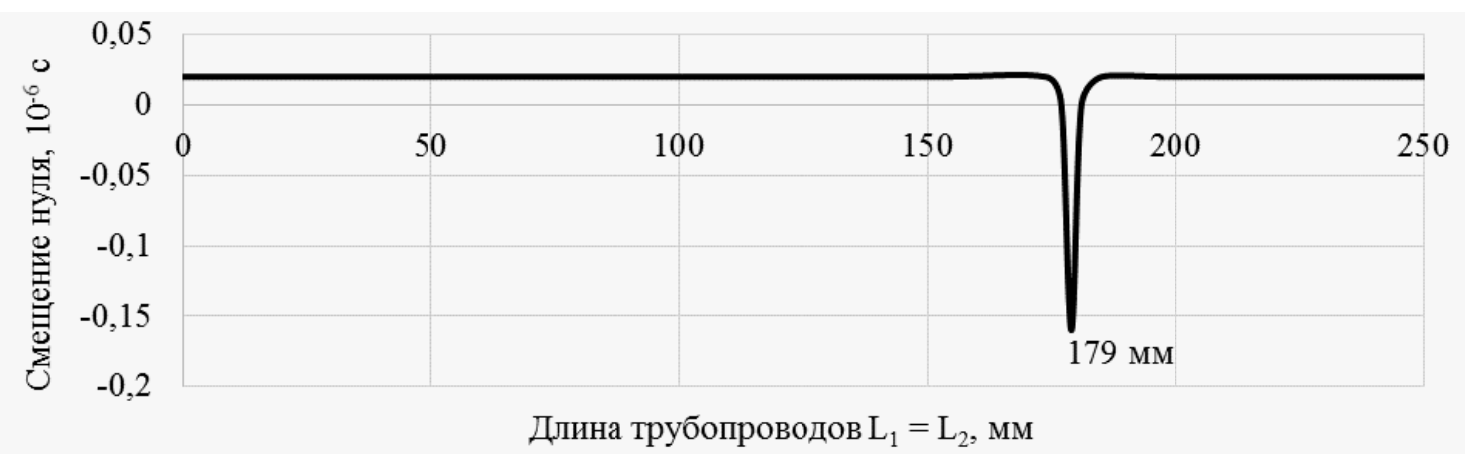

Рис. 10. Зависимость смещения нуля от длины трубопроводов в случае дополнительного закрепления за корпус расходомера со стоячей водой

\section{Заключение}

Изменение жесткости трубопроводов приводит к изменению форм и частот собственных колебаний связанной системы «расходомер-трубопроводы». При сближении частоты собственных колебаний системы «расходомер-трубопроводы» с рабочей частотой резонансных колебаний трубок наблюдается недопустимое смещения нуля.

Проведенные в статье исследования показали, что смещение нуля при неправильном закреплении расходомера может достигать 0,8 мкс, что эквивалентно увеличению погрешности измерений на 5,6 \% (при расходе 1,5 т/ч, равным половине номинального расхода расходомера). Допустимая погрешность расходомера $0,1 \%$. Испытания расходомера с учетом воздействия текущей воды с расходом 1,5 т/ч при том же закреплении показали, что погрешность измерения расхода также составила 5,6\%. Таким образом, чтобы обнаружить эффект недопустимого увеличения погрешности, обусловленного неправильной установкой расходомера, достаточно провести его испытания со стоячей средой.

Как показали наши исследования, уменьшить этот эффект можно, например, за счет закрепления расходомера за корпус. Дополнительное закрепление позволяет уменьшить количество 
форм собственных колебаний системы «расходомер - трубопроводы», частоты которых могут совпасть с рабочей частотой резонансных колебаний расходомера.

Монтаж кориолисового расходомера необходимо вести с учетом частот собственных колебаний системы «расходомер - трубопроводы». В случае сближения собственных частот системы «расходомер - трубопровод» с рабочей частотой резонансных колебаний трубок, с одной стороны, достаточно немного изменить длину трубопровода, чтобы смещение нуля стало минимальным. С другой стороны, рабочая частота резонансных колебаний трубок сильно зависит от плотности текущей внутри трубки среды. Это затрудняет выбор корректной жесткости закрепления расходомера.

На основании полученных результатов исследований считаем необходимым разработать методику учета влияния форм и частот собственных колебаний закрепленного расходомера на смещение нуля с учетом разных плотностей текущей среды внутри трубки.

\section{Испытания проведены с использованием оборудования ЦКП «Экспериментальная механи- ка» ЮУрГУ. \\ Работа выполнена при финансовой поддержке Минобрнауки России. Соглашение о предос- тавлении субсидии № 14.578.21.0191 от 03.10.2016. Уникальный идентификатор соглашения RFMEFI57816X0191.}

\section{Литература / References}

1. Wang T., Baker R. Coriolis Flowmeters: a Review of Developments Over the Past 20 Years, and an Assessment of the State of the Art and Likely Future Directions. Flow Measurement and Instrumentation, 2014, vol. 40, pp. 99-123.

2. Plache K.O. Measuring Mass Flow Using the Coriolis Principle. Loxton R., Pope P. Instrumentation: A Reader, Springer US, 1990, pp. 55-62.

3. Anklina M., Drahmb W., Rieder A. Coriolis Mass Flowmeters: Overview of the Current State of the Art and Latest Research. Flow Measurement and Instrumentation, 2006, vol. 17, pp. 317-323.

4. Raszillier H., Durst F. Coriolis-Effect in Mass Flow Metering. Archive of Applied Mechanics, 1991, vol. 61, no. 3, pp. 192-214.

5. N.M. Keita, Contribution to the Understanding of the Zero Shift Effects in Coriolis Mass Flowmeters. Flow Measurement and Instrumentation, 1989, vol. 1, no. 1, pp. 39-43.

6. Thomsen J.J., Dahl J. Analytical Predictions for Vibration Phase Shifts Along Fluid-Conveying Pipes Due to Coriolis Forces and Imperfections. Journal of Sound and Vibration, 2010, vol. 329, no. 15, pp. 3065-3081.

7. Enz S. Effect of Asymmetric Actuator and Detector Position on Coriolis Flowmeter and Measured Phase Shift. Flow Meas. Instrum. Elsevier Ltd, 2010, vol. 21, no. 4, pp. 497-503.

8. Enz S., Thomsen J.J., Neumeyer S. Experimental Investigation of Zero Phase Shift Effects for Coriolis Flowmeters Due to Pipe Imperfections. Flow Meas. Instrum. Elsevier Ltd, 2011, vol. 22, no. 1, pp. 1-9.

9. Дэссинг О. Испытания конструкций. М.: Брюль и Къер, 1989. 118 с. [Dossing О. Ispytaniya konstruktsiy [Structural Testing, Mechanical Mobility Measurements]. Moscow, Brüel \& Kjæ, Denmark, 1989. 101 p.]

10. Ewins D.J. Modal Testing: Theory, Practice and Application, Hertfordshire. Research Studies Press, 2000. 562 p.

11. Хейлен В., Ламменс С., Сас П. Модальный анализ: теория и испытания. М.: ООО «Новатест», 2010. 319 c. [Heylen W., Lammens S., Sas P. Modal'nyy analiz: teoriya i ispytaniya [Modal Analysis Theory and Testing, Katholieke Universiteit Leuven, Departement Werktuigkunde, 2006.]

12. Maia N.M.M., Silva J.M.M. Theoretical and Experimental Modal Analysis. Research Studies Press, 1997.

13. Peeters B. and et al. The PolyMAX Frequency-Domain Method: a New Standard for Modal Parameter Estimation. Shock and Vibration, 2004, vol. 11, pp. 395-409.

14. Peeters B. and et al. Automotive and Aerospace Applications of the PolyMAX Modal Parameter Estimation. Proceeding of IMAC, 2004, vol. 22, pp. 26-29. 
Яушев Александр Анатольевич, младший научный сотрудник лаборатории «Экспериментальная механика», Южно-Уральский государственный университет, г. Челябинск, iaushevaа@, susu.ru.

Поступила в редакцию 6 ноября 2017 г.

DOI: 10.14529/engin170409

\title{
ANALYSIS OF THE INFLUENCE OF FIXING THE CORIOLIS FLOWMETER ON ZERO POINT SHIFT
}

\author{
A.A. Yaushev, iaushevaa@susu.ru \\ South Ural State University, Chelyabinsk, Russian Federation
}

\begin{abstract}
A Coriolis flowmeter is used for measuring mass flow of liquids and gases. The Coriolis flow meters determine mass flow rate by measuring the phase shift of the U-shaped vibrating tubes. The tubes make forced oscillations at the resonance frequency. It is drive frequency. The phase shift is proportional to the mass flow. Therefore, changes in the phase shift amount not related to the flow of the measured medium, lead to mass flow measurement error. The article is devoted to the experimental evaluation of the effect of mechanical boundary conditions of the Coriolis flowmeter on zero point shift. The zero point shift of the Coriolis flow meter is the value of the phase difference without the flow of the medium. A special equipment, allowing to change the stiffness of the pipeline, to which the flowmeter is attached, has been developed. There have been conducted modal experiments on the Coriolis flowmeter with liquid for different lengths of pipe. Changing the length of the tubing affects the modes of the coupled flowmeter-piping system. The tests were carried out on a flowmeter with tubes filled with water without flow and with a flow of water with a constant flow rate. It has been found out that the maximum zero point shift is observed when the frequency of natural vibrations of the coupled system of the flow meter and piping tubes coincides with the drive frequency of tube vibrations. In the article the additional fixation of the flowmeter for the case. To reduce the modes affecting the zero point shift.

Keywords: Coriolis flowmeter; zero point shift; experimental modal analysis; external vibration; steady forced oscillations.
\end{abstract}

Received 6 November 2017

\section{ОБРАЗЕЦ ЦИТИРОВАНИЯ}

Яушев, А.А. Анализ влияния условий закрепления на смещение нуля кориолисового расходомера / А.А. Яушев // Вестник ЮУрГУ. Серия «Машиностроение». - 2017. - Т. 17, № 4. - С. 91-98. DOI: $10.14529 /$ engin 170409

\section{FOR CITATION}

Yaushev A.A. Analysis of the Influence of Fixing the Coriolis Flowmeter on Zero Point Shift. Bulletin of the South Ural State University. Ser. Mechanical Engineering Industry, 2017, vol. 17, no. 4, pp.91-98. (in Russ.) DOI: 10.14529/engin 170409 